\title{
Doctor-patient communication in a Southeast Asian setting: the conflict between ideal and reality
}

\author{
Mora Claramita • Adi Utarini · Hardyanto Soebono • Jan Van Dalen • \\ Cees Van der Vleuten
}

Received: 13 February 2010/Accepted: 6 June 2010/Published online: 25 July 2010

(C) The Author(s) 2010. This article is published with open access at Springerlink.com

\begin{abstract}
Doctor-patient communication has been extensively studied in non-Western contexts and in relation to patients' cultural and education backgrounds. This study explores the perceived ideal communication style for doctor-patient consultations and the reality of actual practice in a Southeast Asian context. We conducted the study in a teaching hospital in Indonesia, using a qualitative and a quantitative design. In-depth interviews were conducted with ten internal medicine specialists, ten internal medicine residents, 16 patients in two groups based on education level and ten most senior medical students. The contributions of doctors and patients to the communication during consultations were observed and rated quantitatively by thirty internal medicine residents, 393 patients with different educational backgrounds and ten senior medical students. The 'informed and shared decision making' is the central observation in this quantitative study. The results of the interviews showed that Southeast Asian stakeholders are in favor of a partnership style of communication and revealed barriers to achieving this: doctors and patients are not prepared for a participatory style and high patient load due to an inefficient health care system does not allow sufficient time for this type of communication. The results of the quantitative study showed a sharp contrast between observed and ideal communication styles. A paternalistic style seems to prevail, irrespective of patients' educational background. We found a sharp conflict between ideal and reality concerning
\end{abstract}

M. Claramita $(\bowtie)$

The Skills Laboratory, Medical Education Department, Faculty of Medicine, Gadjah Mada University (UGM), Grha Wiyata Building 3rd-Floor, Jalan Farmako Sekip Utara, Yogyakarta 55281, Indonesia e-mail: claramita@yahoo.com

\footnotetext{
A. Utarini

Department of Public Health, Faculty of Medicine, Gadjah Mada University (UGM), Public Health Buidling 2nd Floor, Jalan Farmako Sekip Utara, Yogyakarta 55281, Indonesia

H. Soebono

Department of Dermatology and Venerology, Faculty of Medicine, Gadjah Mada University (UGM), Radiopoetro Building 3rd-Floor, Jalan Farmako Sekip Utara, Yogyakarta 55281, Indonesia

J. Van Dalen · C. Van der Vleuten

School of Health Professions Education, Maastricht University (MU), Maastricht, The Netherlands
} 
doctor-patient communication in a Southeast Asian context. Further studies should examine ways to change the prevailing communication style in the desired direction.

Keywords Doctor-patient communication · Intercultural communication ·

Patients' educational background - The continuum of partnership style

\section{Introduction}

Good doctor-patient communication is essential for positive health care outcomes (Kurtz et al. 2002; Stewart 1984). The partnership communication style presupposes a balanced contribution from doctor and patient (Charles et al. 1999; Makaoul and Clayman 2006). This style ranges between a paternalistic communication style, where the doctor determines the course of the consultation and takes the decisions (Charles et al. 1999) and an extreme consumerism style where the patient dictates what happens during the consultation (Bensing et al. 2006; Charles et al. 1999). The informed and shared decision making between doctors and patients is the substantial issue in the ideal style (Thistlethwaite and Van der Vleuten 2004). However, this style is mainly advocated in Western countries.

In Southeast Asian countries, a different communication style might be more appropriate, because social hierarchy is more prominent and more generally accepted. An important aspect of this strong hierarchy is the respect that is due to someone who is perceived to be of higher social status (e.g. the doctor). Two other traits with relevance to doctor-patient communication in which Southeast Asian societies differ from most Western societies are limited autonomy of the members of a social group, which Hofstede qualifies as collectivism as opposed to individualism, and the use of traditional medicine, which is also influenced by collectivism (Hofstede 2001; Galanti 2008; Moore 2008). These cultural characteristics may foster a one-way or paternalistic communication style, where the doctor, being on a higher hierarchical level, dominates the consultation. It should also be noted here that, in Asian countries, partnership is generally interpreted as an interaction in which the doctor shows a caring attitude and not so much as communication between two parties that are on an equal footing, as is emphasized in Western countries (Moore 2008; Lee et al. 2008).

Doctor-patient communication is further complicated by the educational gap between patients and doctors being generally wider in Southeast Asian societies than in many Western countries. Approximately half of the Indonesian population completes primary education but does not move onto secondary education (BSC-Indonesia 2010). This creates a situation that is not conducive to a partnership style of doctor patient communication (Kim et al. 2003). Studies in Western contexts too have indicated that patients' educational background can influence doctor-patient communication (Murray et al. 2007; Willem et al. 2005). In summary, the type of partnership style communication that is advocated in Western countries may not be entirely suitable in a Southeast Asian context, because of cultural differences and differences in education.

Nevertheless, previous qualitative studies among patients in non-Western countries reported a general preference for a partnership style of doctor-patient communication (Kiguli 2007; Moore 2008). These studies were limited to patients' perceptions, however, and did not investigate the perceptions of other stakeholders nor did they systematically observe communication in real consultations. In order to determine what type of doctorpatient communication style might be most suitable for Southeast Asian countries, it is important to explore which style is preferred by stakeholders and what barriers they 
perceive to stand in the way of the ideal style. In the present study we explored the views of doctors, patients of different education levels and medical students concerning their preferred style of doctor-patient communication and barriers to the use of that style. Additionally, we asked doctors and patients to rate their respective contributions to the communication in a consultation they had just finished, and we asked students who observed the consultations to do the same.

\section{Context}

Our study was conducted in an Indonesian teaching hospital. Most teaching hospitals in Indonesia, including the one where this study was conducted, are general hospitals where primary, secondary and tertiary care is provided, and which thus constitute a rather complex environment.

Indonesia is a country with more than 300 ethnic groups, and it is considered to be historically representative of Southeast Asian society at large (Raffles 2008). Indonesia is in the top four of the most densely populated countries in the world. It covers over 13,000 islands, including five large ones (UN 2008). The teaching hospital where the current study was set is located in Central Java, which is home to eighty percent of the Indonesian population. The ethnic majority of the population is Javanese. Approximately half of the Indonesian population has less than a secondary school education (BSC-Indonesia 2010).

We chose internal medicine as the setting for the study, because it offers case variety and resembles the primary care setting and hence affords optimal observation of doctorpatient communication during consultations (Devito 1995). Internal medicine outpatient clinics in a teaching hospital in Indonesia have specific characteristics: attending internal medicine specialists are only present if necessary, because most of them divide their time between their academic post at the university and clinical work in private hospitals. High patient load is the norm: On a routine day one internal medicine resident will see around thirty patients in the outpatient clinic. Patients have no pre-arranged appointments. Students are present in the teaching hospital but only to observe, they do not actively participate in patient care. Weekly scientific meetings are conducted for internal medicine residents and medical students separately.

\section{Methods}

We used a combined qualitative and quantitative approach with interviews and questionnaires. In-depth interviews with internal medicine residents, patients and students were conducted to explore their views concerning the ideal type of doctor-patient communication in consultations and potential barriers to implementing that style. During the 6 weeks in which the doctors and patients were interviewed, we also conducted a questionnaire survey concerning doctors' and patients' contributions to the communication during real consultations observed by students.

\section{Subjects}

A maximum variation sampling technique was used to compose four groups of stakeholders: 10 internal medicine residents, 10 attending internal medicine specialists, 8 patients who had completed primary school, junior high school or high school (low 
education level), 8 patients who had completed education at a higher level than high school (high education level) and 10 of the most senior medical students. Sampling of participants for the interviews continued until saturation of information was reached. Participation was voluntary and all participants completed a consent form before the interview. No significant problems were encountered in recruiting participants. Those who declined because of personal reasons were replaced by other participants that were comparable in terms of clinical experience (doctors) and education (patients).

In the quantitative part of the study, doctors and patients rated their contributions to the communication in a consultation immediately after the encounter, and the student who observed the consultation did the same. The consultations took place in the outpatient clinic of the teaching hospital. Since internal medicine specialists work in private hospitals, it was not feasible to observe their consultations. All thirty internal medicine residents who ran the outpatient clinic during the study period participated in the study. The 393 patients who participated met the following inclusion criteria: they were able to engage in direct communication with the doctor and they had no very serious condition or terminal illness which could affect their communication style (Deveugele et al. 2002).

The same students who participated in the interviews observed the consultations and rated doctors' and patients' contributions. Since their presence is normal in the outpatient clinic, the students were able to observe unobtrusively. All participants filled in a consent form. The observation study was conducted during a 6-week period, because prolonged observation time was expected to minimize a Hawthorne effect among the doctors. Such an effect can occur when participants in a study behave differently than usual because they know their behavior is a subject of study (Crowl 1996; Streiner and Norman 1995).

Instruments and procedures

The standardized in-depth interviews were guided by key questions. The doctors were asked: "In your opinion, what is the most successful type of doctor-patient communication during a consultation?" and "In your opinion, what are the barriers to achieving that type of communication?" The patients were asked similar questions but framed more indirectly: "What/how do you think the consultation process could contribute maximally to your health outcomes?" and "What/how/which consultation do you think was the most improper/least beneficial doctor-patient communication?"

The interviews were conducted by four interviewers who had been trained by two senior qualitative researchers from the Public Health Department, Faculty of Medicine, Gadjah Mada University, according to a guideline from Utarini (2003). The interviewers attended two sessions of $2 \mathrm{~h}$ each. In the first session they learned interview techniques and during the second session they role played interviews, asking the standardized questions and potential additional ones. After observing the first author conducting the first two interviews with a doctor and a patient, the trained interviewers conducted the remaining interviews. The interviews lasted $\sim 40 \mathrm{~min}$ each and were audio recorded.

A two-item questionnaire was developed to assess communication style. The items asked doctors and patients to rate their respective contributions to the doctor-patient communication during a consultation they had just completed. The rating scales of both items were inspired by the Global Affect Rating explained in The Roter Method of Interaction Process Analysis (Roter 2006 pp. 51), a general affect rating on a six point scale ranging from dominance to interactivity. The Roter Method uses this rating and 41 coding categories for utterances (smallest unit of communication) to analyze interaction processes. We adapted the Global Affect Rating to rate doctors' and patients' contribution to consultations. 


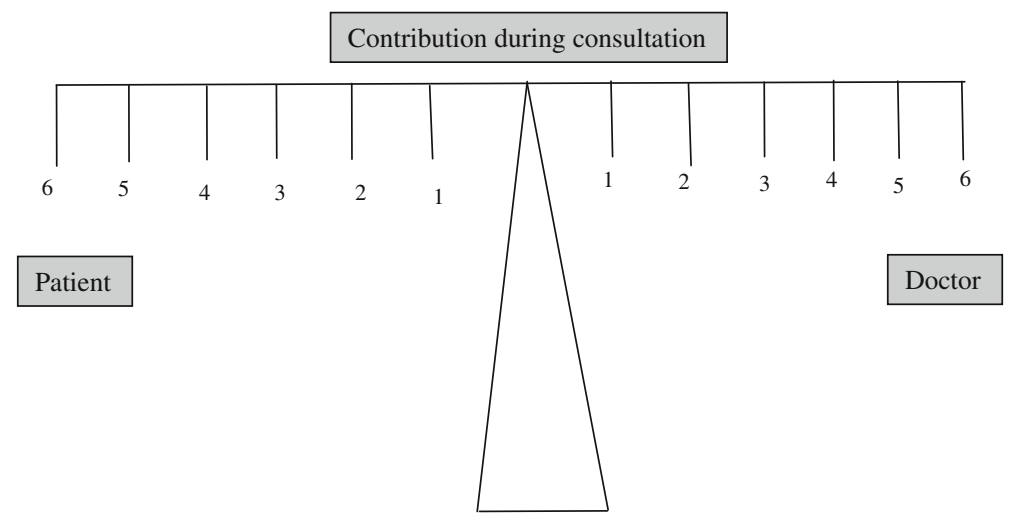

Fig. 1 Visualization of the two questionnaires presented to doctors, patients and students: irrespective contributions to the doctor-patient communication during a consultation in which they participated/ observed. 1 is minimum contribution, 6 is maximum contribution

"Contribution" is a term that doctors and patients in this context naturally interpret as "informed and shared decision making" (Charles et al. 1999; Makaoul and Clayman 2006). It was rated on a six-point scale ( $1=$ minimum contribution; $6=$ maximum contribution). In order to help the participants visualize the balance-imbalance of the contributions of doctor and patient, the scales for the two items were presented visually as one scale balanced symmetrically around the mid-point, representing equal contributions (Fig. 1).

The participating medical students distributed the questionnaires to the participating doctors and patients and were available to answer questions. The thirty participating internal medicine residents rated their first ten to fifteen consultations. This was done in a period of 6 weeks. The 393 participating patients rated their consultation with one of the internal medicine residents and the ten medical students rated each doctor-patient consultation they observed. Rating was done immediately after the consultation in question.

Analysis

The audiotapes of the interviews were transcribed within $48 \mathrm{~h}$ after each interview. A grounded theory approach was used to analyze the transcripts (Finlay and Ballinger 2006; Patton 1990; WHO 1994). The first and second authors individually coded the transcripts into categories and then met regularly to discuss their analysis of the data. This iterative process continued until agreement on the categories and the core category was reached.

Of the questionnaires, fourteen were excluded because they were incomplete, only one item was answered or items were judged by fewer than three raters. Ten further questionnaires were excluded because of very low ratings of the contribution by both the doctor and the patient (both items $\leq 2$ ). With such a low score, it is unlikely that anything significant happened during the consultation. Exclusion of these 24 questionnaires left 369 for analysis. The patients were divided into a high and a low education level group as described above. One-way ANOVA was performed to analyze the data of the questionnaires completed by the unobtrusive observers, but the results were not significant. This might be interpreted as a reflection of the objectivity of the observers in rating both items. We used a t-test for paired samples to compare the doctors', patients' and students' ratings of doctors' and patients' contributions. A critical $p$ value of 0.005 was considered significant. 


\section{Results}

Desired doctor-patient communication style and barriers to achieving the desired style

The analysis of the interviews showed that among the participants there was a general preference for a partnership style of doctor-patient communication. They described a need for mutual understanding in which trust, equality and reciprocal exchange of information and participation featured prominently. Mutual understanding was mentioned by all the respondents in the qualitative study.

"I would like the doctor to understand what I told him and I would like to understand his explanation."

(Patient 3, low education)

"It should be like [thinking...] more between friends."

(Patient 9, high education)

"Good communication results in satisfaction for both parties. Information can then be delivered. Both trust each other."

(Doctor 5, Chief internal medicine resident - Year 5 internal medicine resident)

"The 'sambung rasa' (empathy) of verbal and non verbal communication between a doctor and a patient is the goal."

(Medical student 3)

We identified three major barriers to mutual understanding. The first barrier relates to time constraints that are inherent in the health care system. High patient load does not allow sufficient time for the desired type of communication. This barrier was the first to be identified during the qualitative analysis. Seven of the 10 internal medicine specialists, 9 of the 10 internal medicine residents, all 8 high education level patients, 6 of 8 low education level patients and all 10 students complained that high-patient load influenced the doctorpatient interaction. Time constraints were mentioned explicitly by the participants in the qualitative study.

"Every day for three hours we have to open a clinic for more than 30 patients. It is important to finish consultations as quick as we can."

(Doctor 3: Year 3 internal medicine resident)

"I know the doctor is very busy, he still has long of queue of patients after my turn.

So, I did not want to bother him with too many questions."

(Patient 4: low education)

The second barrier was formed by the fact that patients are not prepared for a participatory communication style. There was a difference between the two groups of patients in this respect. Patients of a high educational level were deemed to be better prepared for a participatory consultation style by 7 of the 10 internal medicine specialists, 6 of the 10 internal medicine residents and 9 of the 10 students. However, patients in the low education level group did not explicitly indicate that they felt unprepared for a participatory communication style. It required careful analysis of indirect remarks to unravel their references to this barrier.

"When the doctor is trying to explain about A, B, C and I do not understand 'B', I ask him 'how's that, Doc?' I don't keep quiet. It is the perfect time to talk about anything and to ask about anything."

(Patient 11, high education) 
"The doctor did not instruct me to get an X-ray again, so I probably should have asked him about the X-ray - but he did not instruct me, so I felt I couldn't ask him that question. The doctor knows more than I do. I think he did the right thing." (Patient 2, low education)

The third barrier was the doctors' lack of the communication skills that are required for a participatory style. During their training, doctors do not learn to use such a communication style during consultations. This barrier was not mentioned by any of the participants, however. It only emerged at the end of the qualitative data analysis. None of the 10 internal medicine specialists talked about this barrier, 2 of the 10 internal medicine residents showed some awareness of it, none of the 10 students spoke directly about doctors' lack of communication skills and none of the patients made any clear reference to this issue. However, critical analysis of the data indicated that doctors' inadequate communication skills could be a barrier.

"I should have listened more...."

(Doctor 1: Year 2 internal medicine resident)

"When the patient replies that he understands, usually a patient with little education,

I end my explanation."

(Doctor 3: Year 4 internal medicine resident)

"I already explained everything. Why does the patient not comply?"

(Doctor 9: Internal medicine specialist, 8 years of experience)

"I have tried to explain many times that I am allergic to a certain drug, I experienced tremors, but the doctor keeps prescribing the same drug again and again....may be the tremors are nothing serious..."

(Patient 3: low education)

"Well, the doctor's response depends on the patient's response. If there is little response, as is usual with patients with lower education, then the doctor will only explain as little as necessary."

(Student 6)

Figure 2 illustrates the desired communication style, the three barriers and how they are interrelated. "High patient load" is the first barrier to the desired mutual understanding because all participants agreed that this is an important impediment to using the ideal communication style. The other two barriers are represented after "high patient load" in an order of importance inferred from the frequency and directness of participants' references to them.

\section{Contributions of doctors and patients to the consultation}

The characteristics of the participants in the quantitative study are presented in Table 1 .

There was no difference between the groups in the distribution of sex and age.

Overall, the doctors' contributions to the communication significantly exceeded those of their patients as judged by all the raters (doctors, patients and students; Table 2).

When doctors communicated with high education patients, the contributions were equal according to both patients and students, but the doctors themselves judged their contributions to be similar with all patients, irrespective of patients' education levels. 


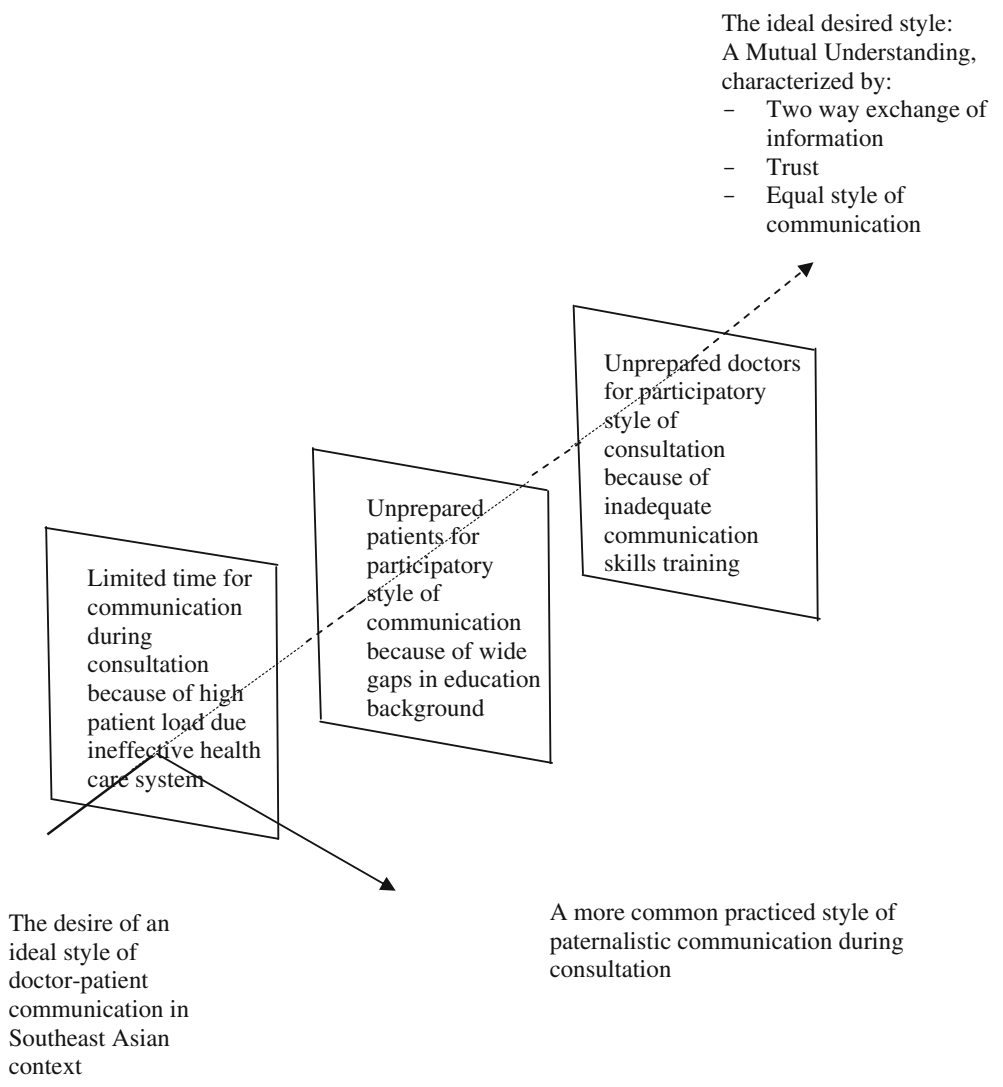

Fig. 2 Barriers in approaching the ideal style of doctor-patient communication during consultation in a Southeast Asian context

\section{Discussion}

The doctors, patients and students that participated in this study uniformly stated that they preferred a partnership communication style. The need for mutual understanding described by the participants is similar to findings reported in studies in Western (Kurtz et al. 2002; Stewart 1984) as well as non-Western settings (Kiguli 2007; Moore 2009). A very striking finding of our study is the marked difference between the expressed preference and what was actually observed during consultations, where communication appeared to be a far cry from the perceived ideal.

Limited time for consultations seems to be the main barrier to the ideal partnership style. The high patient load caused by an ineffective health care system also influences doctorpatient interaction in the teaching hospital. The majority of patients have a low education level and their being unprepared for a participatory style of communication showed in hesitancy to participate during consultations. As a result doctors, who are not trained to use a participatory style, appear to conclude that this style is not required and therefore do not use it. However, an alternative reason why doctors do not use a partnership style may be that in fact they are not equipped to do so. These three barriers together appear to stand in the way of communication aimed at mutual understanding between doctors and patients. 
Table 1 Characteristic of participants of 393 observed consultations

\begin{tabular}{|c|c|c|c|c|}
\hline \multirow[t]{2}{*}{ Participants } & \multirow[t]{2}{*}{ Background } & \multicolumn{2}{|c|}{ Gender } & \multirow{2}{*}{$\begin{array}{l}\text { Age range } \\
\text { (years) }\end{array}$} \\
\hline & & Male & Female & \\
\hline \multicolumn{5}{|l|}{ Doctors } \\
\hline & Year 1 residency & 4 & 4 & $30-34$ \\
\hline & Year 2 residency & 10 & 6 & $28-42$ \\
\hline & Year 3 residency & 3 & 3 & $31-39$ \\
\hline Total & & 17 & 13 & $28-42$ \\
\hline \multicolumn{5}{|l|}{ Patients } \\
\hline & $\begin{array}{l}\text { High education group of patients } \\
\text { (Bachelor degree and above) }\end{array}$ & 50 & 40 & $18-76$ \\
\hline & Low education group of patients & 145 & 158 & $22-84$ \\
\hline & High school graduate & 84 & 89 & $25-81$ \\
\hline & Junior high school graduate & 17 & 18 & $31-78$ \\
\hline & Primary school graduate & 44 & 51 & $27-83$ \\
\hline Total & & 195 & 198 & $18-84$ \\
\hline Students & Most senior students & 4 & 6 & $24-26$ \\
\hline
\end{tabular}

Table 2 Contribution to the communication during consultation as judged by different groups of participants

\begin{tabular}{lllll}
\hline $\begin{array}{l}\text { Contribution to the communication } \\
\text { during a consultation as judged } \\
\text { by different groups of participants }\end{array}$ & $\begin{array}{l}\text { Number of } \\
\text { consultations }\end{array}$ & $\begin{array}{l}\text { Doctors' } \\
\text { contribution } \\
\text { Mean } \pm \text { SD }\end{array}$ & $\begin{array}{l}\text { Patients' } \\
\text { contribution } \\
\text { Mean } \pm \text { SD }\end{array}$ & $\begin{array}{l}p \text { value or } \\
\text { Significance } \\
(t \text {-test paired } \\
\text { sample })\end{array}$ \\
\hline Overall & 369 & $4.02 \pm 1.08$ & $3.69 \pm 1.12$ & $p<0.01$ \\
Doctors & 369 & $4.26 \pm 0.95$ & $3.82 \pm 1.14$ & $p<0.01$ \\
Patients & 369 & $4.63 \pm 1.09$ & $4.32 \pm 1.12$ & $p<0.01$ \\
Students & 369 & $4.06 \pm 0.84$ & $3.71 \pm 1.09$ & $p<0.01$ \\
High education group-patients & & & & $p<0.01$ \\
Overall & 84 & $4.03 \pm 1.25$ & $3.76 \pm 1.26$ & $p<0.01$ \\
Doctors & 84 & $4.42 \pm 0.94$ & $3.98 \pm 1.04$ & $p<0.01$ \\
Patients & 84 & $4.53 \pm 1.04$ & $4.30 \pm 1.10$ & Not significant \\
Students & 84 & $4.14 \pm 0.81$ & $3.88 \pm 0.99$ & Not significant \\
Low education group-patients & & & & $p<0.01$ \\
Overall & 285 & $4.03 \pm 1.03$ & $3.68 \pm 1.09$ & $p<0.01$ \\
Doctors & 285 & $4.21 \pm 0.95$ & $3.81 \pm 1.17$ & $p<0.01$ \\
Patients & 285 & $4.67 \pm 1.10$ & $4.33 \pm 1.26$ & $p<0.01$ \\
Students & 285 & $4.04 \pm 0.85$ & $3.67 \pm 1.12$ & $p<0.01$ \\
\hline
\end{tabular}

In a study on doctor-patient communication that was conducted in a family planning setting in Southeast Asia (Kim et al. 2003), it was also suggested that low education might explain why patients seem unprepared for a participatory consultation style. Strong social hierarchy was put forward as a culture-related explanation in another study, but this study provided limited in-depth analysis (Moore 2008). Both studies also referred, in passing, to 
the high patient load in Asian contexts. Our study confirmed that education and patient load impede the use of a partnership style, and added a third barrier: doctors are not well prepared for using this style. Thus doctors' inadequate communication training could be one of the keys to the prevailing usage of a paternalistic communication style during consultations.

According to the patients with high education, their contribution to the consultation was equal to that of the doctor. This perception was confirmed by the students but not by the doctors, who considered their contribution to be largest with both groups of patients. More highly educated patients may experience difficulties during consultations because their view of the communication style is different to that of their doctor. Less highly educated patients may not feel offended by the doctor's larger contribution, since they concur with the doctor's view: doctors contribute more to consultations than patients. Studies conducted in Western countries have shown that patients with higher levels of education tend to expect a partnership style of communication, whereas patients with lower educational levels tend to elicit a more paternalistic style (Murray et al. 2007; Willem et al. 2005). In the present study, education level was associated with differences in patients' perceptions of the communication in real consultations whereas doctors thought they used the same paternalistic style with all patients.

This study was limited to an Internal Medicine setting. We do not know how representative this context is of other disciplines. All ten students who participated in the in-depth interview also witnessed the 393 rated consultations. Therefore their participation in both methods may influence their opinion in the interview and their rating on doctorpatient consultations. Another limitation is that only residents were observed, because specialists work in private hospitals. This situation is inherent in the nature of the health care system. However, when we observed consultations in private practice we found a comparable situation: long queues of patients in the waiting rooms and a minimum number of previously arranged appointments. These observations were not part of this study. We actually observed each of 3 internal medicine specialists from another small town during 10 consultations in a private practice setting and 10 consultations of the same three internal medicine specialists in a primary care setting and found that the results in both setting were very similar to those of the present study. However, only a limited number of specialists work in both settings, so we can only speculate about this. The forth limitation is that we were unable to recruit a patient sample with an even distribution of educational levels, probably because patients with higher education tend to prefer a private hospital to a teaching or public hospital.

We think that the results of our study in a single hospital in a Southeast Asian country may be generalized to the whole Southeast Asian context. We understand that Indonesia and other Southeast Asian countries have many ethnic groups, but, in our view, these ethnic groups are very similar in terms of strong social hierarchy and less emphasis on individual autonomy than on community. Decision making and the use of traditional medicine are influenced by the importance of non-verbal expression in communication and the communal system (Hofstede 2001; Galanti 2008). We therefore believe that the results of this study are also representative of doctor-patient communication in other Southeast Asian contexts.

In theory, the three barriers that emerge from this study can be overcome by effective communication skills training for doctors. However, efforts to achieve a desired style of doctor-patient communication are always rely on a particular context and culture (Rees and Sheard 2002). Not only is it very complicated to influence the organization of the health care system, it is likely impossible to do so in the current context. Important in this 
respect is the phenomenon that the culture seems to rely more strongly on wishes than on scheduled and structured plans for putting ideas into practice. We cited a study of a fundamental Anthropologist (Geertz 1976) who did ethnography research at a specific region in Southeast Asia:

"...the slametan (the ceremony of wishes), form a kind of social universal joint, fitting the various aspect of social-life and individual experience together in way which minimizes uncertainty, tension and conflict or at least it is supposed to do so." "Slametan can be given in response to almost any occurrence of one wish to celebrate, ameliorate, or sanctify (from birth, marriage, sorcery, death, house moving, opening factory, up to political meetings)."

(Geertz 1976, Chapter 1 page 11)

Geertz showed that instead of planning in a systematic and structured way, people in a small region of Southeast Asia perform series of ceremonial wishes to their God, in order to avoid any problem in the future. As a consequence, it might be difficult to restructure the present health care system into a more structured and planned systems where patient load can be reduced to manageable numbers and doctor-patient relationship may obtain adequate time for consultation. It will be quite a challenge to introduce a more systematic primary health care system. Nonetheless, this is today's reality in Southeast Asia. Further studies should examine ways to promote the desired style of doctor patient communication and examine the impact of cultural factors on communication. However, the conflict between the ideal situation and actual practice is probably very typical of a Southeast Asian context.

Acknowledgment We would like to acknowledge the doctors and patients and students in this study who have been willing to participate to finding evidence in the area of doctor-patient communication. We are thankful to Marijke Kruithof for fruitful discourses, Mickey Chenault and Nawi Ng for statistical review and Mereke Gorsira for assisting in the English. This paper is supported by The NPT Project 2008, the Netherlands Government cooperation between UGM and UM.

\section{Conflict of interest None.}

Ethical approval This study had been approved by The Committee of Ethics Faculty of Medicine Gadjah Mada University, Yogyakarta on 7 November 2007 on the document no: KE/FK/251/EC.

Open Access This article is distributed under the terms of the Creative Commons Attribution Noncommercial License which permits any noncommercial use, distribution, and reproduction in any medium, provided the original author(s) and source are credited.

\section{References}

Bensing, J. M., Tromp, F., Van Dulmen, S., Van den Brink-Muinen, A., Verheul, W., \& Schellevis, F. G. (2006). Shifts in doctor-patient communication between 1986 and 2002: A study of videotaped General Practice consultations with hypertension patients. BMC Family Practice, 7, 62.

Board Statistic Centre of Indonesia. (2010). Indonesian labor situation. Official Statistic. No. 33/05/Th. XIII, 10 Mei 2010.

Charles, C., Whelan, T., \& Gafni, A. (1999). What do we mean by partnership in making decisions about treatment? BMJ, 319, 780-782.

Crowl, T. K. (1996). Fundamentals of educational research. New York: McGraw-Hill.

Deveugele, M., Derese, A., \& De Maeseneer, J. (2002). Is GP-patient communication related to their perceptions of illness severity, coping and social support? Social Science and Medicine, 55, 1245-1253. 
Devito, J. A. (1995). The interpersonal communication handbook (7th ed.). NY: Harper Collins College Pub. Finlay, L., \& Ballinger, C. (2006). Qualitative research for allied health professionals; challenging choices. West Sussex: Wiley.

Galanti, G. A. (2008). Caring for patients (pp. 93-108). Philadelphia: University of Pennsylvania Press. pp. $197-221$.

Geertz, C. (1976). The religion of Java. Chicago and London: The University Chicago Press.

Hofstede, G. (2001). Culture's consequences, comparing values, behaviors, institutions, and organizations across nations. Newbury Park, CA: Sage Publications.

Kiguli, S. (2007). The expectations and experiences of caregivers of pediatric patients admitted at Mulago hospital regarding the communication skills of the attending doctors. A Thesis in partial fulfillment for the award of the degree of Masters of Health Professions Education. University of Maastricht. The Netherlands. Maastricht.

Kim, Y. M., Putjuk, F., Basuki, E., \& Kool, A. (2003). Increasing patient participation in reproductive health consultation: An evaluation of smart patient coaching in Indonesia. Patient Education and Counseling, $50,113-122$.

Kurtz, S., Silverman, J., \& Draper, J. (2002). Teaching and learning communication skills in medicine. Oxon: Radcliffe Medical Press.

Lee, K. H., Seuw, A., Luo, N., \& Koh, D. (2008). Attitudes towards the doctor-patient relationship. Medical Education, 42(11), 1092-1099.

Makaoul, G., \& Clayman, M. L. (2006). An integrative model of shared decision making in medical encounters. Patient Education and Counseling, 60, 301-312.

Moore, M. (2008). What does patient-centered communication mean in Nepal? Medical Education, 42, $18-26$.

Moore, M. (2009). What do Nepalese medical students and doctors think about patient-centred communication? Patient Education and Counseling, 76, 38-43.

Murray, E., Pollack, L., White, M., \& Lo, B. (2007). Clinical decision making: patients' preferences and experiences. Patient Education and Counseling, 65, 186-196.

Patton, M. Q. (1990). Qualitative evaluation and research method (2nd ed.). London: Sage Publication.

Raffles, T. H. (2008). The history of Java. Yogyakarta: Narasi Publication.

Rees, C., \& Sheard, C. (2002). The relationship between medical students' attitude towards communication skills learning of students with their demographic and education-related characteristic. Medical Education, 36, 1017-1027.

Roter, D. (2006). The Roter method of interaction process analysis. Baltimore: The John Hopkins University. Bloomberg: School of Public Health. Department of Health Policy and Management.

Stewart, M. A. (1984). What is a successful doctor-patient interview? A study of interactions and outcomes. Social Science Medicine, 19, 167-175.

Streiner, D. L., \& Norman, G. R. (1995). Health measurement scales. A practical guide to their development and use. Oxford: Oxford University Press.

Thistlethwaite, J. E., \& Van der Vleuten, C. (2004). Informed shared decision making: view and competencies of pre-registration house officers in hospital and general practice. Education for Primary Care, 15, 83-92.

United Nations. (2008). World population prospects. Department of Economic and Social Affairs Population Division.

Utarini, A. (2003). Module of qualitative research for postgraduate education. Yogyakarta: Public Health Department Faculty of Medicine Gadjah Mada University.

Willem, S., de Maesschalcks, S., Deveugele, M., Derese, A., \& de Maeseneer, J. (2005). Socio-economic status of the patient and doctor-patient communication: Does it make a difference? Patient Education and Counseling, 56, 139-146.

World Health Organization. (1994). Qualitative research for health programs. Geneva: WHO Division of Mental Health. 